\title{
Improving children emergency care satisfaction through standardized approach of pediatric triage
}

\author{
Sahar Mahmoud El-Khedr Abd El-Gawad \\ Department of Pediatric Nursing, Faculty of Nursing, Tanta University, Tanta, Egypt
}

\section{Email address:}

Sahar.khedr@yahoo.com

\section{To cite this article:}

Sahar Mahmoud El-Khedr Abd El-Gawad. Improving Children Emergency Care Satisfaction through Standardized Approach of Pediatric Triage. American Journal of Nursing Science .Vol. 3, No. 6, 2014, pp. 91-99. doi: 10.11648/j.ajns.20140306.11

\begin{abstract}
Triage is the process of determining the priority of emergency patients' treatments based on the severity of their condition. Pediatric triage assessment is a rapid, focused evaluation to gather pertinent subjective and objective data to determine the severity of illness. This can be achieved through standardized triage approach. Patient satisfaction is considered an important indicator of the quality of care from the perspective of the consumer. Satisfied patients are more likely to comply with treatment, take an active role in their own care. The study aims to improve children emergency care satisfaction through standardized approach of pediatric triage. This is a quasi-experimental study design, which was carried out at Pediatric Emergency Department at Al-Menshawy General Hospital, Tanta City. Purposive sample of one hundred children were involved in the study. They were divided into two groups' fifty children in each one. Data was collected from the period of June to the end of September 2013. Two tools were used to collect data, the first tool was a questionnaire sheet used to collect socio-demographic data. The second tool was a Consumer Emergency Satisfaction Scale (CECSS). The results of this study revealed that, $60 \%$ of the triage group was satisfied by emergency visit compared to $26 \%$ of the non-triage group. There was a statistical significant difference regarding total nursing care and total education between the triage and non-triage group. It is recommended to apply triage nursing care at all Emergency Departments.
\end{abstract}

Keywords: Emergency Care, Satisfaction, Standardized Approach, Pediatric Triage

\section{Introduction}

Satisfaction can be defined as the extent of an individual's experience compared with his or her expectations. Evaluating to what extent patients are satisfied with health services is clinically relevant, as satisfied patients are more likely to comply with treatment, take an active role in their own care, to continue using medical care services, stay within a health provider and maintain with a specific system. In addition, health professionals may benefit from satisfaction surveys that identify potential areas for service improvement and health expenditure may be optimized through patient-guided planning and evaluation ${ }^{(1)}$

Satisfaction is not some pre-existing phenomenon waiting to be measured, but a judgment people form over time as they reflect on their experience. Patients' satisfaction with health care they received becomes a priority issue. Satisfied patients are more likely to seek and comply with prescribed treatment regimes. ${ }^{(2)}$ Patients participation is increasingly being linked with improvements in the quality of health care and improved health outcomes. Patient satisfaction is considered an important indicator of quality care from the perspective of the consumer and has been widely studied in many settings. However, few studies have examined patient satisfaction with emergency nursing services in the particular area of triage. ${ }^{(3)}$

The word "triage" comes from the French word trier, which means to sort or to select. Its historic roots for medical purposes go back to the days of Napoleon probably around 1792, when triaging large groups of wounded soldiers was necessary. An effective triage system helps nurses to ensure that patients seeking emergency care "receive appropriate attention, in a suitable location, with the requisite degree of urgency" and that emergency care is initiated in response to clinical need rather than order of arrival. Triage refers to sorting or sifting. ${ }^{(4,5)}$ Triage is the process of determining the priority of emergency patients treatments based on the severity of their condition and priority of emergency 
transport. Triage aims to promote the safety of patients by ensuring that timing of care and resource allocation is requisite to the degree of illness or injury. An effective triage system classifies patients into groups according to acuity of illness or injury and aims to ensure that the patients with life threatening illness or injury receive immediate intervention and greatest resource allocation. Triage is an ongoing process involving continuous assessment and reassessment ${ }^{(6)}$.

Triage nurses must also be able to discriminate useful cues from large amounts of information in order to perform triage safely. It is the responsibility of the triage nurse to rapidly identify and respond to actual life-threatening states and to also make a judgment as to the potential for life-threatening states to occur. The allocation of a triage category is made on the basis of necessity for time-critical intervention to improve patient outcome, potential threat to life or need to relieve suffering. The decisions made by a triage nurse are a pivotal factor in the initiation of emergency care. Therefore the accuracy of triage decisions is a major influence on the health outcomes of patients ${ }^{(7,8)}$.

Triage decisions can be divided into primary and secondary decisions. Primary triage decisions relate to the assessment, allocation of a triage category and patient deposition whilst secondary triage decisions relate to the initiation of nursing interventions in order to expedite emergency care and promote patient comfort. The triage nurse is the first person that a patient encounters when presenting for emergency care. The triage nurse should be highly skilled in interpersonal and communication skills. She has a responsibility to be polite, professional and reassuring whilst eliciting the information he or she requires making a triage decision. ${ }^{(9)}$ Patients and their families should have access to information regarding the triage process. This information should include a simple explanation of the principles of triage, the triage categories, how the patient has been categorized and their intended waiting time. The reason for delays in waiting times, in case of the presence of seriously ill or injured patients. ${ }^{(10)}$

Emergency care continues to be a challenge for the pediatric population. Pediatric patients who seek emergency care require skilled and timely assessments by experienced emergency nurses. The pediatric triage assessment is a rapid, focused 3 to 5 minutes evaluation of the pediatric patient that gathers pertinent subjective and objective data to determine the severity of illness. ${ }^{(11)}$ It is important to have a reliable triage system in place that allows for rapid and accurate assessment of patients. Nationwide, there are an estimated 30 million ED visits per year for patients under 18 years of age, accounting for one-fourth of all ED visits. ${ }^{(8)}$ Children's physiological and psychological responses to stressors are not the same as those of adults, and they are more susceptible to a range of injuries and illnesses. ${ }^{(12)}$

Standardized approach of triage is a formulation, and implementation of guidelines, rules and specification of action for common and repeated use. It aims to achieve optimum care in triage emergency care. The goal of the triage nurse is to rapidly and accurately assess an ill child in order to assign a triage level to guide timely routing to the appropriate emergency department area for definitive evaluation and management. Triage nurse should keep in mind the use of a standardized approach to triage assessment of the pediatric patient. ${ }^{(13)}$ Once the patient arrives in the ED, the triage process begins. The patients are sorted or classified according to their presenting symptoms. The stable patient is directed to obtain specific demographic information about the patient and initiates the written or electronic record. The patient is then directed to be evaluated. Stable patients are sent to the triage nurse, who then completes the remainder of the triage assessment. Unstable patients are taken directly back to a treatment room for advanced care. ${ }^{(14)}$

Because of the potential for rapid deterioration of the pediatric patient, a precise and accurate triage process is critical. The comprehensive triage process supports a rapid assessment and prioritization of patients performed by an educated and experienced pediatric nurse. The comprehensive triage process includes evaluation and classification of all incoming patients to the ED, initiation of first aid measures, ordering diagnostic studies and procedures according to prescribed triage protocols. ${ }^{(15,16)}$ More recently, a five-level system has been suggested for pediatric patients which is similar to the adult five-level acuity classifications; critical, emergent, urgent, non-urgent, and fast track. Many of the specific pediatric triage systems are based on the primary survey of Airway, Breathing, and Circulation (ABCs) $(15)$

\section{Significance of the Study}

Emergency admissions are rising and bed crises are occurring almost daily in many hospitals. Children requiring emergency care have unique and special needs. Emergency care continues to be a challenge for the pediatric patients. It is one of the most sensitive areas of health care. This sensitivity is commonly based on a combination of factors such as urgency and crowding. The aim of triage is to improve the quality of emergency care and prioritize cases according to urgency. Effective triage system classifies patients according to acuity of illness or injury and providing immediate intervention and greatest resource allocation. In addition, patient dissatisfaction due to increased waiting time for transfer to an inpatient bed has also become the most important cause of pediatric emergency department dissatisfaction.

\section{Aim of the Study}

This study aims to improve children emergency care satisfaction through standardized approach of pediatric triage.

Hypothesis:

- Standardized approach of pediatric triage enhances emergency nursing care.

- Children are more satisfied with emergency nursing care using triage system.

- There is no significant difference in the quality of care 
provided by nurses to both triage and non-triage group. Subjects and Methods

A-Subjects

Design:

A quasi-experimental design was used in the current study.

Setting: Pediatric Emergency Department at Al-Menshawy General Hospital, Tanta City.

Sampling:

A purposive sample of 100 children was drawn from the Emergency Department over a period of four months from the beginning of June to the end of September 2013. Children were divided into two groups 50 Children in each group. The first group is a non-triage group who received the routine emergency nursing care that was provided according to hospital policy. The second group was a triage group who received emergency care using the standardized approach of pediatric triage.

Inclusion criteria:

1 Age ranged from 12-18 years.

2 Free from any communication or psychological problems

Tools: Two tools were used in this study. The first tool was a questionnaire sheet that was developed by the researchers to collect socio-demographic data about the children involved in this study; it included age, gender, presence of pain and chronic illness. The second tool was Consumer Emergency Care Satisfaction Scale (CECSS). The CECSS was used to examine patient satisfaction with the use of triage nursing care. This tool was divided into two subscales; these were titled caring (12 items) and Teaching ( 3 items). The remaining four items were all negatively worded filter items to minimize bias, which was included in the tool but, excluded from the scoring.

The CECSS was classified into five-point Likert-type rating scale demonstrating the degree of satisfaction with each statement. Possible responses ranged from strongly disagree (1) to strongly agree (5). A higher score represents a higher degree of satisfaction with emergency nursing. A total score of more than $60 \%$ indicated satisfaction, while the score less than $60 \%$ indicated dissatisfaction ${ }^{(17)}$. A pilot study was conducted on $10 \%$ of patients minor modifications were made to the questionnaires. The content validity of a tool was reviewed by a panel of experts who rate the relevance of each item in the tool and assessed the whole tool. Cronbach's alpha coefficient of 0.97 for the Caring subscale, and 0.88 for the Teaching subscale.

\section{Data Collection}

Interview was conducted with children and simple explanation about the aim of the study was done for children and their accompanying care giver. Those who agreed to participate were involved in the study. They completed the questionnaire before their medical consultation to ensure that the satisfaction scores obtained were related to the triage nursing care and no other nursing care received in the emergency department.
B-Methods:

The researcher collects socio-demographic data from children using a questionnaire through individualized interview. Children' satisfaction of the non-triage group were assessed using CECSS before discharge and after receiving routine emergency care according to hospital policy. CECSS was used to assess satisfaction of the triage group after applying triage nursing care. The children were classified into five levels which are; level one-critical and need resuscitation; level two-high risk, emergent; level threemoderate risk, urgent; level four-low risk, semi-urgent; and level 5-lower risk, non-urgent. ${ }^{(18)}$ Emergency Severity Index (ESI) with the triage group. It was used as a standardized approach of pediatric triage. ESI was 6 step approach that aims to provide rapidly and accurately assessment of ill children in order to assign a triage level for definitive evaluation and management. ${ }^{(19,20)}$ According to ESI, children in the triage group were classified through 6 steps as follow:

Step 1. Appearance/breathing/circulation (ABCD) "quick assessment": The researcher assessed children' appearance from tone, inter-activeness, consolability, look, and speech or cry. The work of breathing was assessed through observation of the airway sounds, positioning, retractions, and flaring. Circulation to skin was assessed by observing pallor, mottling, or cyanosis. The nurse can get a quick idea of the physiological stability of a child and, in conjunction with the chief complaint, make decisions regarding the need for life support. Some children may need to be taken immediately to the treatment area to address abnormalities found in the quick assessment. For more stable children, the researcher proceeds to the next step in the assessment.

Step 2. Airway/breathing/circulation/disability/exposureenvironmental control (ABCDE): This assessment includes assessing for airway patency, respiratory rate and quality, heart rate, skin temperature and capillary refill time, blood pressure, and an assessment for disability or neurological status. A child's neurological status, level of consciousness, and pupillary reaction. Exposure involves undressing the patient to assess for injury or illness, and addressing any immediate environmental needs such as treating fever. Any serious finding in the $\mathrm{ABCDE}$ assessment indicates a need for immediate treatment.

Step 3. Pertinent history: a standardized history was obtained that included the onset of symptoms, allergies, associated symptoms, treatment prior to arrival.

Step 4. Vital signs: The researcher assess vital signs and if needed oxygen saturation.

Step 5. Fever:

Step 6. Pain: Pain assessment was assessed using a validated pediatric pain scale. It includes assessment of Pain, Quality, Radiation, Location, \& Severity (PQRLS). Children who meet the $\geq 7$ criterion was considered for triage as an ESI level 2, and should use sound clinical judgment in making the final decision ${ }^{(19)}$ 


\subsection{Pilot Study}

Prior to the actual study, a pilot study was conducted on $10 \%$ of the study sample to test study tools for its clarity, feasibility, applicability and determine the required time to fulfill the tools and then necessary modifications was done. The pilot study was excluded from the research.

\subsection{Ethical Consideration}

A formal approval was taken from the administrator of AlMenshawy general Hospital and the head of the Emergency unit. Simple explanation about the aim of the study was also done for the care givers and their children and those how accepted to participate were involved in the current study. During the work every effort was made to ensure the safety, privacy, and confidentiality for each child.

\subsection{Statistical Analysis}

The collected data were organized, tabulated, computerized and analyzed using SPSS (17). It includes percentage, mean, and t-test, $\chi 2$. Correlation test were also done.

\subsection{Limitation of the Study}

Limited space and overcrowding in the emergency department causes the triage nurse to under triage a patient, and patients who seen were sent to the waiting room. Training for the emergency nurses who were willing to participate in the study take time and effort to ensure that they can apply triage correctly.

\section{Results}

Table 1. Distribution of children according to demographic data $(n .=50)$.

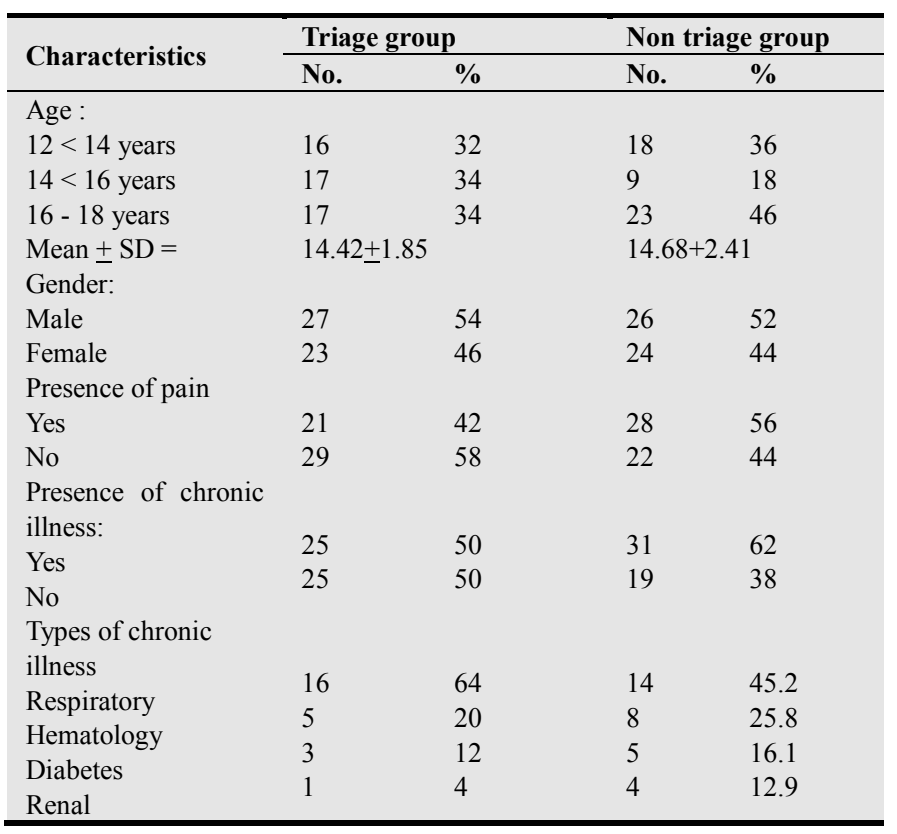

Regarding children's demographic data, it was clear that the mean age of children was $14.42 \pm 1.85$ in triage group while it was $14.68+2.41$ in non-triage group. Slightly more than half of the sample (54\%) and (52\%) of both triage and non-triage group respectively were males. Forty two and $56 \%$ of triage and non-triage group respectively had pain. Presence of chronic illness was reported by half of the triage group and $52 \%$ of the non-triage group. Slightly less than two thirds of the children who has chronic illness in the triage group complained of respiratory disease. While it was $45.2 \%$ of the non-triage group. (Table 1 )

Table (2) shows distribution of children according to transportation, disposition received nursing care. It was regarded that, the entire triage group $(100 \%)$ arrived to hospital by car/self-compared to $96 \%$ of the non-triage group. Eighty percent of the children in the triage group and $74 \%$ of the non-triage group discharged after receiving emergency care. Nearly equal percent $64 \%$ and $66 \%$ of the triage and non-triage group respectively received base line assessment while, $36 \%$ of the triage group and $34 \%$ of the non-triage group received specific intervention.

Figure (1) shows waiting time as reported by children. It was clear that, $58 \%$ of the triage group waiting less than 15 minutes. Thirty two percent and $16 \%$ of the triage and nontriage group respectively were waiting from 30-45 minutes. Slightly more than half of the non-triage group (52\%) waited from 45-60 minutes compared to $8 \%$ of the triage group. Statistical significant difference was found $(\mathrm{P}=.000)$.

Triage group classification according to Emergency Severity Index (ESI) showed that $36 \%$ of the children in the triage group were non-urgent cases compared to $40 \%$ who were semi-urgent, $16 \%$ urgent and $8 \%$ emergent. None of the triage group was classified as critical case. Figure (2).

Table 2. Distribution of children according to transportation, disposition and received nursing care $(n .=50)$.

\begin{tabular}{llllc}
\hline \multirow{2}{*}{ Items } & \multicolumn{2}{c}{ Triage group } & \multicolumn{2}{c}{ Non triage group } \\
\cline { 2 - 5 } & $\mathbf{N}$. & $\mathbf{\%}$ & $\mathbf{N}$. & $\%$ \\
\hline Arrival Transport & 50 & 100 & 48 & 96 \\
Car/Self & - & - & 2 & 4 \\
Ambulance & 40 & 80 & 37 & 74 \\
Disposition & 10 & 20 & 13 & 26 \\
Discharged & & & & \\
Admitted & 32 & 64 & 33 & 66 \\
Received nursing care & 18 & 36 & 17 & 34 \\
Base line assessment & & & & \\
Specific intervention & &
\end{tabular}

Table (3) presents children satisfaction regarding nursing care. It was evident that, $82 \%$ of the triage group reported that, the nurse knows something about their illness compared to $50 \%$ of the non-triage group and there was statistical significant difference. Equal percent $72 \%$ of the triage group mentioned that the nurses were gentle when performing painful procedure, seemed to understand their feeling and take time to meet their needs. Forty eight, $28 \%$ and $44 \%$ of the children in the non-triage group reported that the nurse were gentle, understand and meet their needs respectively. Statistical significant difference were found in most of the items and over all nursing care $(\mathrm{P}=.000)$.

Children' satisfaction about nursing education was 
illustrated in table (4). It was clear that, $68 \%$ and $34 \%$ of the triage and non-triage group reported that nurses instruct them about home self-care and there was statistical significant difference. Fifty four percent \& $34 \%$ of the triage and nontriage group mentioned that nurses told them about their problem. The triage group reported that nurses told them what to expect at home $68 \%$ compared to $36 \%$ of non-triage group. Statistical significant difference was found regarding overall nursing education $(\mathrm{P}=.000)$.

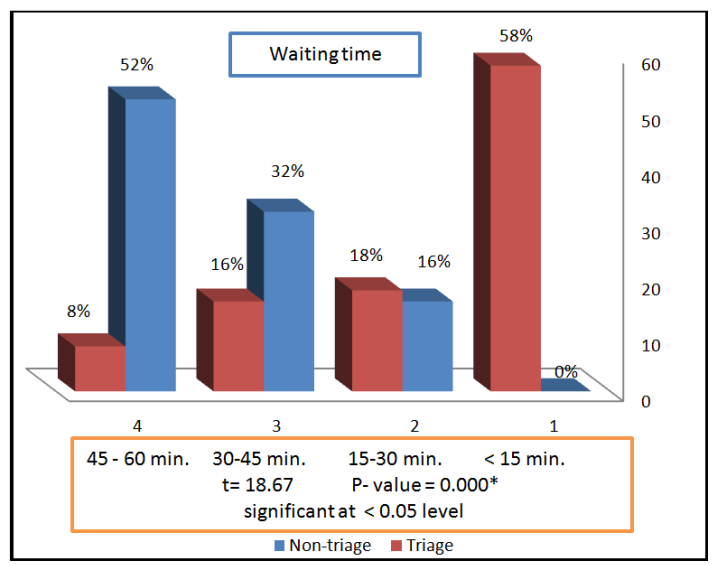

Figure (1). Shows distribution of children according to waiting time.

As regards, table (5) Satisfaction about nurses and general environment. It was clear that, nearly one third of the triage group (32\%) mentioned that the nurses were genuine compared to only $10 \%$ of the non-triage group. Thirty four percent and $32 \%$ of the triage group reported that, the nurses were efficient and respect privacy compared to equal percent of $14 \%$ of the non-triage group who reported the same response. Nearly one quarter of the triage group (24\%) and $40 \%$ mentioned that, the nurse welcomed them on admission very good and good respectively compared to $20 \%$ and $30 \%$ of the non-triage group respectively. Twenty two percent and $32 \%$ of the triage group reported that the environment was comfortable and quite respectively, while it was $30 \%$ and $20 \%$ of the non-triage group and there was statistical significant differences were found regarding satisfaction with environment ( $\mathrm{P}=.002)$.

As regards overall patients' satisfaction about emergency experiences. Table (6) shows that $60 \%$ of the triage group reported that their emergency visit was good compared to $26 \%$ of the non-triage group and there was statistical significant difference. Equal percent (38\%) of both triage and non-triage group were satisfied by services provided in the emergency department. Twenty six percent and $46 \%$ of triage and nontriage group respectively reported satisfaction with environment and there was statistical significant difference. Slightly more than two thirds of the triage group mentioned the need to increase the number of staff to enhance emergency compared to $36 \%$ of the non-triage group. Statistical significant difference was found regarding the efforts to enhance emergency.

Table (7) shows the correlations between triage group' satisfaction of total care and total education and their sociodemographic characteristics and waiting time. It was evident that, there were significant correlations between patients' age and both pain and illness, Significant correlations also, found between total care and total education and waiting time. Significant correlations also were found between total education and pain and, between total nursing care, and patients' illness.

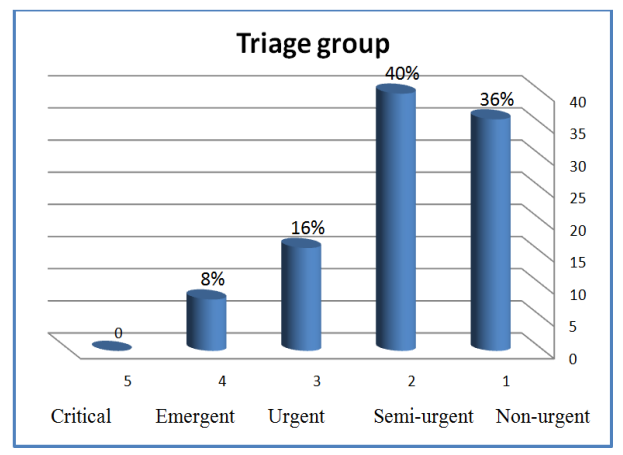

Figure (2). Shows distribution of triage group according to Emergency Severity Index.

Table 3. Distribution of children according to satisfaction about nursing care $(n .=50)$.

\begin{tabular}{|c|c|c|c|c|c|c|c|}
\hline \multirow{2}{*}{\multicolumn{2}{|c|}{ Nursing care statements: The nurse }} & \multirow{2}{*}{\multicolumn{2}{|c|}{$\begin{array}{l}\text { Triage group } \\
\text { Satisfied } \\
\end{array}$}} & \multirow{2}{*}{\multicolumn{2}{|c|}{$\begin{array}{l}\text { Non-triage group } \\
\text { Satisfied } \\
\end{array}$}} & \multirow{3}{*}{$\chi^{2}$} & \multirow{3}{*}{ P value } \\
\hline & & & & & & & \\
\hline & & $\mathbf{N}$. & $\%$ & N. & $\%$ & & \\
\hline 1 & performed her duties with skill & 28 & 56 & 25 & 50 & 3.670 & .055 \\
\hline 2 & know something about my illness & 41 & 82 & 25 & 50 & 174.92 & $.000^{*}$ \\
\hline 3 & knew what treatment I needed & 32 & 64 & 22 & 44 & 2.536 & .111 \\
\hline 4 & explained procedures before they were done & 27 & 54 & 26 & 52 & 68.66 & $.000^{*}$ \\
\hline 5 & explained things in terms I could understand & 31 & 62 & 19 & 38 & .512 & .474 \\
\hline 6 & understand when listening to my problem & 34 & 68 & 25 & 50 & .810 & .368 \\
\hline 7 & seemed genuinely concerned about my pain, & 26 & 52 & 23 & 46 & 27.453 & $.000^{*}$ \\
\hline 8 & was gentle when performing painful skill & 36 & 72 & 24 & 48 & 6.100 & .014 \\
\hline 9 & seemed to understand how I felt & 36 & 72 & 14 & 28 & 46.995 & $.000^{*}$ \\
\hline 10 & gave me a chance to ask questions & 31 & 62 & 21 & 42 & 6.100 & $.014 *$ \\
\hline 11 & appeared to take time to meet my needs & 36 & 72 & 22 & 44 & 46.995 & $.000^{*}$ \\
\hline 12 & sure that all my questions were answered & 32 & 64 & 26 & 52 & 14.155 & $.000^{*}$ \\
\hline \multicolumn{2}{|c|}{ Overall nursing care } & & & & & 531.82 & $0.000 *$ \\
\hline
\end{tabular}

N.B. Table shows satisfaction responses only.

*Significant at $\leq 0.05$ level 
Table 4. Distribution of children according to satisfaction about nursing education ( $n .=50)$.

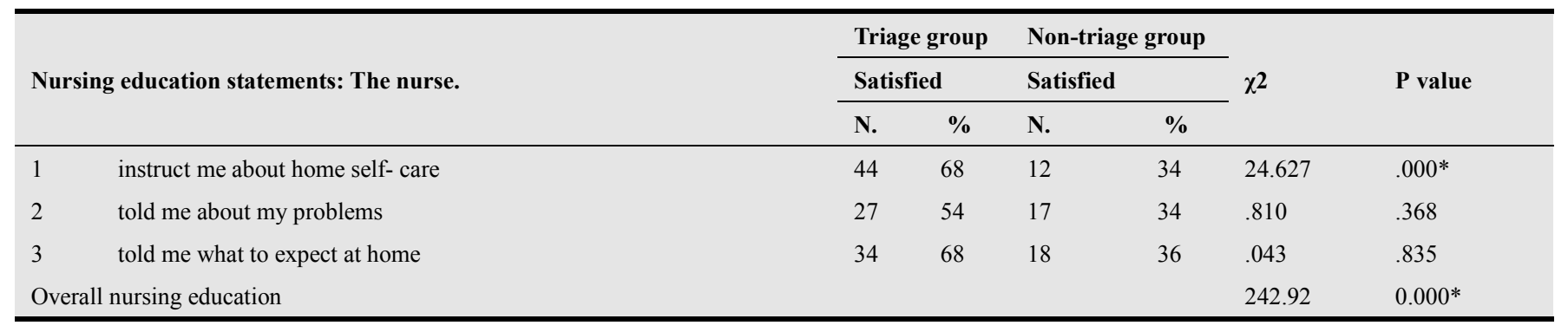

N.B. Table shows satisfaction responses only.

*Significant at $\leq 0.05$ level.

Table 5. Distribution of children according to satisfaction about nurses and general environment $(n .=50)$.

\begin{tabular}{|c|c|c|c|c|c|c|}
\hline \multirow{3}{*}{ Items } & \multirow{2}{*}{\multicolumn{2}{|c|}{$\begin{array}{l}\text { Triage group } \\
\text { Satisfied }\end{array}$}} & \multicolumn{2}{|c|}{ Non triage group } & \multirow{3}{*}{$\chi^{2}$} & \multirow{3}{*}{ P value } \\
\hline & & & \multicolumn{2}{|c|}{ Satisfied } & & \\
\hline & No. & $\%$ & No. & $\%$ & & \\
\hline \multicolumn{7}{|c|}{ 1-Personal Qualities of Nurse: } \\
\hline Caring & 13 & 26 & 11 & 22 & & \\
\hline Helpful & 11 & 22 & 11 & 22 & 3.60 & 0.308 \\
\hline Calm & 10 & 20 & 23 & 46 & & \\
\hline Genuine & 16 & 32 & 5 & 10 & & \\
\hline \multicolumn{7}{|c|}{ 2-Professional Qualities of Nurse: } \\
\hline Efficient, & 17 & 34 & 7 & 14 & & \\
\hline Knowledgeable & 8 & 16 & 10 & 20 & 6.16 & 0.104 \\
\hline Privacy respected & 16 & 32 & 7 & 14 & & \\
\hline Treated everyone equally & 9 & 18 & 26 & 52 & & \\
\hline \multicolumn{7}{|c|}{ 3-Nurses welcomed the patients: } \\
\hline Very Good & 12 & 24 & 10 & 20 & & \\
\hline Good & 20 & 40 & 15 & 30 & 5.360 & 0.147 \\
\hline $\mathrm{Bad}$ & 12 & 24 & 9 & 18 & & \\
\hline Nothing & 6 & 12 & 16 & 32 & & \\
\hline \multicolumn{7}{|l|}{ 4-Environment: } \\
\hline Comfortable & 11 & 22 & 15 & 30 & & \\
\hline Quiet & 16 & 32 & 10 & 20 & 16.80 & $0.002 *$ \\
\hline Disturbing & 9 & 18 & 13 & 26 & & \\
\hline Clean & 14 & 28 & 12 & 24 & & \\
\hline
\end{tabular}

Table shows positive responses only.

*Significant at $\leq 0.05$ level.

Table 6. Distribution of children' overall satisfaction about their emergency experience $(n .=50)$.

\begin{tabular}{|c|c|c|c|c|c|c|}
\hline \multirow{3}{*}{ Satisfaction about ER experience } & \multicolumn{2}{|c|}{ Triage Group } & \multicolumn{2}{|c|}{ Non triage group } & \multirow{3}{*}{$\chi^{2}$} & \multirow{3}{*}{ P value } \\
\hline & \multicolumn{2}{|c|}{ Satisfied } & \multicolumn{2}{|c|}{ Satisfied } & & \\
\hline & No. & $\%$ & No. & $\%$ & & \\
\hline \multicolumn{7}{|l|}{ 1) Emergency visit } \\
\hline Good & 30 & 60 & 13 & 26 & \multirow{3}{*}{6.02} & \multirow{3}{*}{$0.049^{*}$} \\
\hline Fair & 12 & 24 & 22 & 44 & & \\
\hline Poor & 8 & 16 & 15 & 30 & & \\
\hline \multicolumn{7}{|l|}{ 2) What did you like in ER? } \\
\hline Service & 19 & 38 & 19 & 38 & \multirow{4}{*}{23.12} & \multirow{4}{*}{$0.000^{*}$} \\
\hline Environment & 13 & 26 & 23 & 46 & & \\
\hline Nurse behaviour & 10 & 20 & 3 & 6 & & \\
\hline Flexibility & 8 & 16 & 5 & 10 & & \\
\hline \multicolumn{7}{|l|}{ 3) Efforts to enhance emergency } \\
\hline Increase number of staff & 34 & 68 & 18 & 36 & \multirow{4}{*}{43.04} & \multirow{4}{*}{$0.000 *$} \\
\hline Large Wating room & 18 & 18 & 13 & 26 & & \\
\hline Increase number of equipment & 8 & 8 & 14 & 28 & & \\
\hline Presence of doctors all time & 6 & 6 & 5 & 10 & & \\
\hline
\end{tabular}

N.B. Table shows positive responses only.

*Significant at $\leq 0.05$ level. 
Table 7. Correlation matrix between triage group' satisfaction with total nursing care, total education and their demographic characteristics and waiting time.

\begin{tabular}{|c|c|c|c|c|c|c|c|c|}
\hline Items & & Age & Gender & Pain & Illness & Waiting & Total care & Total education \\
\hline Age & - & - & & & & & & \\
\hline \multirow{2}{*}{ Gender } & $\mathrm{r}$ & 0.058 & - & & & & & \\
\hline & $\mathrm{P}$ & 0.568 & - & & & & & \\
\hline \multirow{2}{*}{ Pain } & $\mathrm{r}$ & 0.225 & 0.162 & - & & & & \\
\hline & $\mathrm{P}$ & $0.025^{*}$ & 0.108 & - & & & & \\
\hline \multirow{2}{*}{ Illness } & $\mathrm{r}$ & 0.255 & $-0.106-$ & $-0.098-$ & - & & & \\
\hline & $\mathrm{P}$ & $0.010^{*}$ & 0.295 & 0.330 & - & & & \\
\hline \multirow{2}{*}{ Waiting } & $\mathrm{r}$ & 0.010 & $-0.205-$ & $-0.075-$ & $-.186-$ & - & & \\
\hline & $\mathrm{P}$ & 0.925 & $0.041^{*}$ & 0.458 & .064 & - & & \\
\hline \multirow{2}{*}{ Total care } & $\mathrm{r}$ & 0.134 & 0.145 & 0.052 & $-.248-$ & 0.411 & - & \\
\hline & $\mathrm{P}$ & 0.182 & 0.150 & 0.604 & $.013^{*}$ & $0.000^{* *}$ & - & \\
\hline \multirow{2}{*}{$\begin{array}{l}\text { Total } \\
\text { education }\end{array}$} & $\mathrm{r}$ & 0.014 & 0.023 & $-0.231-$ & .141 & 0.209 & 0.130 & - \\
\hline & $P$ & 0.890 & 0.821 & $0.021^{*}$ & 0.161 & $0.037^{*}$ & 0.196 & - \\
\hline
\end{tabular}

* Correlation is significant at the 0.05 level (2-tailed).

** Correlation is significant at the 0.01 level (2-tailed)

\section{Discussion}

Triage is the process of determining the priority of emergency patients' treatments based on the severity of their condition. The pediatric triage assessment is a rapid, focused evaluation to gather subjective and objective data to determine the severity of illness. The goal of the triage nurse is to rapidly and accurately assess an ill child in order to assign a triage level to guide timely routing to the appropriate emergency department area for definitive evaluation and management. Patient satisfaction is an attitude a person's general orientation towards a total experience of health care. Satisfaction comprises both cognitive and emotional facets and relates to previous experiences, expectations and social networks. Patient satisfaction should form part of continuous improvement. It is a method of evaluating the quality of health services provided to patients. Patient satisfactions with emergency nursing services in the particular area of triage need further studies. This study aims to improve emergency care satisfaction through standardized approach of pediatric triage.

The present study revealed that slightly more than one half of the patients in both triage and non-triage group were male. Statistical significant difference was found regarding the mean of waiting time between the triage and non-triage group. This may be explained by the fact that sorting and classifying patients in the emergency department helping the nurse to determine the priority of care. Nurses also are able to provide immediate nursing intervention according to the hospital policy. All of the actions help in decreasing the waiting time and length of stay in emergency department.

This finding is congruent with the study of Choi YF etal., (2006) how found that triage system improve the waiting time and processing time in busy EDs without additional staff. This improvement reflect improved efficiency of patient processing. ${ }^{(21)}$ The results of this study contradict with the result of Harding K.A (2012) who reported that, the triage process ensured rapid service for a small number of urgent referrals, but made little difference to the waiting time of the vast majority of patients. $^{(22)}$

Statistical significant differences were found between triage and non-triage group regarding the nursing care. Children' satisfaction regarding nursing care was found in two thirds of the triage group and half of the non-triage group. Slightly less than three quarters of the triage group were reported that the nurses were gentle and understanding compared to slightly half and nearly one quarter of the nontriage group. This difference in children satisfaction may be occurred because; triage nurses explain and discuss important interventions with children and their care givers during their assessment. The nurses also, directed the patients throughout the emergency visit and also gave them health teaching and immediate care.

These findings are consistent with the study of Daniel I. (2012) who explored patient satisfaction with nursing care variable, answer, explain, trust, respect, courtesy and availability. These findings support the importance of the interpersonal aspect of nursing practice. ${ }^{(23)}$ The current study findings are also consistent with previous studies that found patients expect the following nursing qualities: friendly, kind, quick to respond to patients' needs, and having adequate time to provide care. Kane et al. (2007) ${ }^{(24)}$

The findings of the current study revealed that, children' characteristics, such as age, gender, presence of pain and previous illness have been found to be related to their satisfaction of total care and education. These results are congruent with the findings of Bacon \& Mark, (2009) who reported that there is a correlation between patients' satisfaction and cultural background, age, sex, and education. ${ }^{(25)}$ Other studies did not find any relationships between patient satisfaction and demographic variables Laschinger et al., (2011) ${ }^{(26)}$. Patients' satisfaction in the present study was correlated to waiting time, as reported in the results of this study, decrease in waiting time in the triage group leading to increase of the patients' satisfaction with nursing care provided. 


\section{Conclusion}

Children were generally satisfied with the care provided by the triage nurses. Measuring children' satisfaction with triage nursing care remains a major challenge for health care providers in emergency care settings. Triage has become an integral part of the function of pediatric emergency nurses, and has demonstrated clinical and organizational value. There is a significant difference regarding overall children' satisfaction with total nursing care and total education. Statistical significance correlation was found between the total care, total education and age, presence of pain and waiting time.

\section{Recommendations}

1 Application of nursing triage in all emergency departments.

2 Developing an educational program for nurses working in non-triage hospitals about the application of triage system in Emergency Department.

3 Develop educational program for nurses about interpersonal skills, work autonomously and make precise decision based on the focused assessment.

4 Enforcing patients' satisfaction as an indicator of the quality of nursing care provided in Emergency Department.

5 Further studies are needed to evaluate the effectiveness of triage nursing care in decreasing the health hazards resulting from delaying in nursing / medical interventions.

\section{Acknowledgements}

The researcher is grateful to all emergency nurses and medical staff who assisted in the current research.

\section{Reference}

[1] Lari M.A., Tamburini M. and Gray D., Patients' needs, satisfaction, and health related quality of life: Towards a comprehensive model, Health and Quality of Life Outcomes 2004; 2:32. http://www.hqlo.com.

[2] Taylor, C. \& Benger, J.R. Patient satisfaction in Emergency Medicine. Emergency Medicine Journal 2004; 12(3): 528-532.

[3] Michael P. Simple tips to improve patient satisfaction, American Academy of Emergency Medicine, 2011;18(1):1819

[4] Robertson S, Evolution of triage systems, Emerge Med J. February2006; 23(2): 154-155.

[5] George S, Read S, Westlake L, Williams B, Pritty P, Fraser Moodie A. Nurse triage in theory and in practice. Archives of Emergency Medicine 1993; 10(3):220-8.

[6] Australasian College for Emergency Medicine. Guidelines for implementation of the Australasian Triage Scale in Emergency Departments.

http://www.acem.org.au/open/documents/triageguide.htm ed, 2000.
[7] Merriam-Webster Online Dictionary Retrieved from $\mathrm{http}: / / w w w . m e r r i a m-w e b s t e r . c o m / d i c t i o n a r y /$ triage, 2008;1205.

[8] Whitby S, Ieraci S, Johnson D, Mohsin M. Analysis of the process of triage: the use and outcome of the National Triage Scale. Liverpool: Liverpool Health Service, 1997.

[9] David W. Warren, MD; Anna Jarvis, MD., Louise LeBlanc, RN., Jocelyn Gravel, MD, and the CTAS National Working Group (NWG) Revisions to the Canadian Triage and Acuity Scale Pediatric Guidelines (Paed.CTAS), CJEM 2008;10(3):224-32.

[10] Kathleen A. O'Neill, RN, MSN, and Kenneth Molczan, RN, MSN, Pediatric Triage: A 2-Tier, 5-Level System in the United States, Pediatric Emergency Care , Lippincott Williams \& Wilkins, August 2003;19(4).

[11] Ojanen Thomas D. Special considerations for pediatric triage in the Emergency Department. Nurs Clin North Am. 2002; $37: 145-159$.

[12] Middleton DR, Burt CW., Availability of pediatric services and equipment in emergency departments: United States, 2002-2003. Advance Data 2006; 367:1-16.

[13] Use of the ESI for Pediatric Triage, Emergency Severity Index (ESI) Implementation Handbook, Agency for healthcare research and quality, 2012 , chapter $6, w w w . h h s . g o v$.

[14] Hohenhaus SM, Travers D, Mecham N. Pediatric triage: a review of emergency education literature. JEN 2008; 34(4):308-313.

[15] Travers D, Agans R, Eitel D, Mecham N, Rosenau A, Tanabe P. Waller A. Reliability evaluation of the Emergency Severity Index Version 4 [Abstract]. Acad Emerge Med 2006; 13(5S):S126.

[16] Gravel J, Gouin S, Bailey B, et al. Reliability of a computerized, version of the Pediatric Canadian Triage and Acuity Scale. Acad. Emerge Med 2007; 14:864-9.

[17] Davis, B.A., Bush, H.A. and Thomas, S.W. Measuring consumer satisfaction with emergency department nursing care. Journal of Nursing Science1997; 2(1-2):35-47.

[18] Briggs, J. K., \& Grossman, V. Emergency nursing 5-tier triage protocols. Philadelphia: Lippincott Williams \& Wilkins, 2006.

[19] Travers D, Waller A, Katznelson J, Agans R. Reliability and validity of the Emergency Severity Index for pediatric triage. Acad Emerge Med 2009; 16(9):843-849.

[20] Rockville, MD., Emergency Severity Index (ESI) Implementation Handbook, 2012 Edition: Chapter 6. Use of the ESI for Pediatric Triage. November 2011. Agency for Healthcare Research and Quality, http://www.ahrq.gov/professionals/systems/hospital/esi/esi6.ht $m l$

[21] Y F Choi, $\mathrm{T}$ W Wong, and $\mathrm{C}$ Lau, Triage rapid initial assessment by doctor (TRIAD) improves waiting time and processing time of the emergency department, Emerge Med J. April 2006; 23(4): 262-265.

[22] Harding KE, Taylor NF, Leggat SG, and Stafford M., Effect of triage on waiting time for community rehabilitation: a prospective cohort study. Arch Phys Med Rehabil. 2012; 93(3):441-5. 
[23] Daniel I, the Relationship between Nurse Staffing and Patient Satisfaction in Emergency Departments, Institute of Health Policy, Management and Evaluation University of Toronto, 2012.

[24] Kane, R. L., Shamliyan, T., Mueller, C., Duval, S. \& Wilt, T. J. "Nursing Staffing and Quality of Patient Care." Evidence Report/Technology Assessment No. 151 Agency for Healthcare Research and Quality2, 007; 1-115.
[25] Bacon, C. T. \& Mark, B."Organizational effects on patient satisfaction in hospital medical-surgical units." Journal of Nursing Administration 2009; 39: 220-227.

[26] Laschinger, H., Gilbert, S. \& Smith, L. "Nursing Outcomes; the State of the Science." Jones \& Barlett, Learning, 2011, ISBN 978-0-7637-8325-9. 